\title{
Joint Synchronization, Channel Length Estimation, and Channel Estimation for the Maximum Likelihood Sequence Estimator for High Speed Wireless Communications
}

\author{
Jyh-Hau Chen \\ Yumin Lee \\ Graduate Institute of Communication Eng. and \\ Dept. of Electrical Engineering, \\ National Taiwan University \\ Taipei 10617, TAIWAN
}

\begin{abstract}
The performance of channel-estimation-based maximum likelihood sequence estimator (MLSE) depends on the accuracy of the channel estimate. Conventional least-squares channel estimators preset a fixed length for the channel. For wireless communications, however, the actual length of the channel is environment-dependent and in general unknown. It is therefore desirable to modify conventional channel estimation algorithms so that the length of the channel estimator adapts to the actual channel. In this paper, we propose a least-squares algorithm for joint synchronization, channel length estimation, and channel estimation. Simulation results show that the proposed scheme is robust against propagation environment and achieves the best trade-off between performance and complexity.
\end{abstract}

\section{INTRODUCTION}

High-speed wireless data communications encounter delay-spread multipath propagation. Adverse effects of multipath propagation include inter-symbol interference (ISI) and signal fading. ISI refers to the interference between successive received symbols, and occurs when the transmission symbol rate is high so that the wireless propagation channel exhibits frequency-selectivity. It significantly limits the performance of a wireless communication system because an irreducible bit error rate (BER) floor results if no mitigation mechanism is employed. Signal fading, on the other hand, is associated with the rapidly time-varying nature of the wireless propagation channel, which is in turn caused by mobility, environmental changes, and/or carrier frequency changes.

Equalization is an effective measure against ISI in wireless data communication systems. A broad class of equalizers, known as channel-estimation-based equalizers[1-4], requires an estimate of the discrete-time equivalent channel in order to function properly. The performance of these equalizers has been analyzed in the literature [4-9] and shown to be sensitive to the quality of the channel estimate. For channel-estimation-based equalizers, the discrete-time equivalent channel is first estimated. The equalizer is then optimized based on the channel estimate. The performance naturally depends on the accuracy of the channel estimates. Conventional channel estimation algorithms preset a fixed length for the channel. For wireless communications, however, the actual length of the channel is environment-dependent. Therefore, unless the receiver has explicit knowledge of the propagation environment, the actual length of the wireless channel is unknown. The effect of mismatch between the length of the channel estimator and actual length of the discrete-time equivalent channel has also been investigated in the literature [9]. It has been shown that an irreducible BER floor results when the channel estimator length is shorter than the actual channel [9]. On the other hand, if the channel estimator length is longer than the actual channel, the equalizer becomes unnecessarily complex. Furthermore, a performance loss that increases with the channel estimator length is still incurred [9].

Although various methods are available in the literature for accurate estimation of the channel of a known length, very little results are available for the estimation of the length of the channel. It is therefore desirable to modify conventional channel estimation algorithms so that the length of the channel estimator adapts to the actual channel. In this paper, we propose a least-squares approach for joint synchronization, channel length estimation, and channel estimation. Simulation results show that the proposed scheme is very robust against variations of the propagation environment and achieves the best trade-off between performance and complexity.

\section{System Model}

The baseband-equivalent system model used throughout our simulations consists of the digital transmitter, wireless channel, and receiver, as shown in Figure 1. The transmitter is implemented according to the physical layer specifications of General Packet Radio Service (GPRS) [10]. Specifically, a random binary data stream with equally likely zeros and 


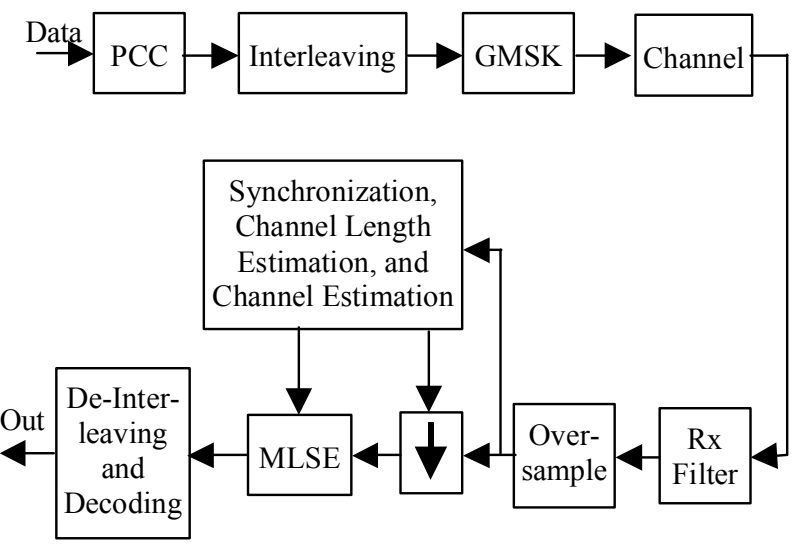

Figure 1. The system block diagram of this paper.

is generated and channel-coded using rate $1 / 2$ punctured convolutional codes with various puncturing patterns. The coded bits are then interleaved and mapped onto GPRS time slots of length 148 symbol-periods. The modulation is Gaussian minimum shift keying (GMSK) modulation [11] with $\mathrm{BT}=0.3$, where $\mathrm{B}$ is the $3-\mathrm{dB}$ bandwidth of the Gaussian filter and $T$ is the symbol period and is set to $48 / 13 \mu \mathrm{s}$. The baseband-equivalent modulated signal $u(t)$ is transmitted through the wireless channel, which is modeled as a frequency-selective multipath fading channel corrupted by additive white Gaussian noise (AWGN) according to the GPRS specifications. The base-band equivalent output of the channel is given by

$$
y(t) \equiv(u \otimes h)(t)+z(t)),
$$

where $h(\bullet)$ is the baseband-equivalent impulse response of the wireless channel, and $z(\bullet)$ is the complex AWGN.

At the receiver, the received signal is first filtered using a square-root raised cosine filter $50 \%$ with roll-off. The output signal of the receiver filter is next sampled at a rate of $\mathrm{L}$ samples per symbol-period. Sampling instant optimization, channel length estimation, and channel estimation are then performed jointly using the algorithm proposed in this paper. The over-sampled signal is then down-sampled to yield a symbol-spaced discrete-time signal. Although GMSK is a nonlinear modulation, the symbol-spaced discrete-time signal can still be approximated [12] as

$$
r_{k}(\tau) \equiv y(k T+\tau)=\sum_{j=0}^{v} b_{k-j} p_{j}(\tau)+z_{k}(\tau),
$$

where $\tau$ is the "optimal" sampling instant, $b_{k}$ are the modulation symbols, $p_{j}(\tau), j=0,1, \ldots, v$ is the discrete-time equivalent channel corresponding to the sampling instant $\tau$, the integer $v$ is the channel memory, and $z_{k}(\tau)$ are samples of the Gaussian noise at the output of the receiver filter. The signal $r_{k}(\tau)$ is processed by a symbol-spaced maximum next performed using standard Viterbi algorithm to obtain the information bits.

\section{Joint Synchronization, Channel Length Estimation, AND CHANNEL Estimation}

In this paper, it is assumed that the optimal channel estimator memory $v^{\prime}$ is an integer between $v_{\min }$ and $v_{\max }$ (inclusive). Furthermore, it is assumed that coarse timing recovery has been performed, so that the desired value for the sampling instant $\tau$ is searched only over a finite set $\Lambda$. The proposed method for joint synchronization, channel length estimation, and channel estimation is performed under these assumptions based on the least-squares channel estimation error metric. Mathematically, let $\hat{p}_{j}\left(v^{\prime}, \tau\right), j=0,1, \cdots v^{\prime}$ be the linear least-squares channel estimate with memory $v^{\prime}$ and corresponding to sampling instant $\tau$. The least-squares channel estimate can be expressed using matrix-vector notation as [13]

$$
\hat{\mathbf{p}}\left(v^{\prime}, \tau\right) \equiv\left[\begin{array}{c}
\hat{p}_{0}\left(v^{\prime}, \tau\right) \\
\vdots \\
\hat{p}_{v^{\prime}}\left(v^{\prime}, \tau\right)
\end{array}\right]=\left(\mathbf{X}_{v^{\prime}}^{H} \mathbf{X}_{v^{\prime}}\right)^{-1} \mathbf{X}_{v^{\prime}}^{H} \mathbf{r}(\tau),
$$

where

$$
\begin{aligned}
\mathbf{r}(\tau) & \equiv\left[\begin{array}{c}
r_{K+Q-1}(\tau) \\
\vdots \\
r_{K}(\tau)
\end{array}\right], \\
\mathbf{X}_{v^{\prime}} & \equiv\left[\begin{array}{ccc}
b_{K+Q-1} & \cdots & b_{K+Q-v^{\prime}-1} \\
\vdots & & \vdots \\
b_{K} & \cdots & b_{K-v^{\prime}}
\end{array}\right],
\end{aligned}
$$

$Q$ is the observation interval, and $b_{K-v^{\prime}}, \ldots, b_{K+Q-1}$ are the training symbols. The corresponding channel estimation error is given by

$$
\begin{aligned}
\varepsilon\left(v^{\prime}, \tau\right) & =\left\|\mathbf{r}(\tau)-\mathbf{X}_{v^{\prime}}\left(\mathbf{X}_{v^{\prime}}^{H} \mathbf{X}_{v^{\prime}}\right)^{-1} \mathbf{X}_{v^{\prime}}^{H} \mathbf{r}(\tau)\right\|^{2} \\
& =\left(\|\mathbf{r}(\tau)\|^{2}-\left\|\mathbf{X}_{v^{\prime}}\left(\mathbf{X}_{v^{\prime}}^{H} \mathbf{X}_{v^{\prime}}\right)^{-1} \mathbf{X}_{v^{\prime}}^{H} \mathbf{r}(\tau)\right\|^{2}\right)^{\prime}
\end{aligned}
$$

where $\|\bullet\|$ denotes the two-norm of a vector. The proposed method uses $\varepsilon\left(v^{\prime}, \tau\right)$ as a basis for the joint optimization of $v^{\prime}$ and $\tau$. The linear least-squares channel estimates corresponding to the optimal $v^{\prime}$ and $\tau$ is then used in the MLSE.

The proposed algorithm for jointly optimizing $v^{\prime}$ and $\tau$ is as follows. The second term in the parenthesis of (6) can be recognized as the projection or $\mathbf{r}(\tau)$ on the column space of $\mathbf{X}_{v^{\prime}}$. Denoting this projection as $\hat{\mathbf{r}}_{v^{\prime}}(\tau)$, we have the following recursive relationship:

$$
\left\|\hat{\mathbf{r}}_{v^{\prime}+1}(\tau)\right\|^{2}=\left\|\hat{\mathbf{r}}_{v^{\prime}}(\tau)\right\|^{2}+\left|\hat{\mathbf{x}}_{v^{\prime}+1}^{H} \mathbf{r}(\tau)\right|^{2}
$$

where 


$$
\hat{\mathbf{x}}_{v^{\prime}+1} \equiv \frac{\mathbf{x}_{v^{\prime}+1}-\mathbf{X}_{v^{\prime}}\left(\mathbf{X}_{v^{\prime}}^{H} \mathbf{X}_{v^{\prime}}\right)^{-1} \mathbf{X}_{v^{\prime}}^{H} \mathbf{x}_{v^{\prime}+1}}{\left\|\mathbf{x}_{v^{\prime}+1}-\mathbf{X}_{v^{\prime}}\left(\mathbf{X}_{v^{\prime}}^{H} \mathbf{X}_{v^{\prime}}\right)^{-1} \mathbf{X}_{v^{\prime}}^{H} \mathbf{x}_{v^{\prime}+1}\right\|},
$$

in which $\mathbf{x}_{v^{\prime}+1}$ is the last column of $\mathbf{X}_{v^{\prime}+1}$. Note that the quantity in (7) is the orthogonal projection, normalized to have unity norm, of the last column of $\mathbf{X}_{v^{\prime}+1}$ on the orthogonal complement of the column space of $\mathbf{X}_{v^{\prime}}$. This quantity depends only on the training symbols and can be pre-computed and stored. Substituting (7) into (6), we have

$$
\varepsilon\left(v^{\prime}+1, \tau\right)=\varepsilon\left(v^{\prime}, \tau\right)-\left|\hat{\mathbf{x}}_{v^{\prime}+1}^{H} \mathbf{r}(\tau)\right|^{2} .
$$

It can therefore be seen that for fixed $\tau, \varepsilon\left(v^{\prime}, \tau\right)$ can be computed recursively. Making use of (8), the proposed algorithm is follows:

Initialization

1. Set $v_{0}=v_{\min }$, and compute $\varepsilon\left(v_{0}, \tau\right)$ for all $\tau \in \Lambda$ using (6).

2. Let $\tau_{\mathrm{o}}=\operatorname{argmin} \varepsilon\left(\nu_{0}, \tau\right)$ and $\varepsilon_{\min }=\varepsilon\left(\nu_{0}, \tau_{0}\right)$, where the minimization is over $\tau \in \Lambda$.

For $v^{\prime}=v_{\min }+1 \ldots v_{\max }$ repeat the following

3. Compute $\varepsilon\left(v^{\prime}, \tau\right)$ for all $\tau \in \Lambda$ from previous values using (6).

4. If $\varepsilon_{\min } /\left(\min \varepsilon\left(v^{\prime}, \tau\right)\right)>\lambda$, where the minimization is over $\tau \in \Lambda$ and $\lambda>0$ is a pre-determined threshold, then perform the following:

(a) Set $v_{0}=v^{\prime}$

(b) Set $\tau_{0}=\operatorname{argmin} \varepsilon\left(v_{0}, \tau\right)$

(c) Set $\varepsilon_{\min }=\varepsilon\left(v_{0}, \tau_{0}\right)$.

The final values of $v_{0}$ and $\tau_{0}$ are then used for computing the least-squares estimates $\hat{p}_{j}\left(v_{\mathrm{o}}, \tau_{\mathrm{o}}\right), j=0,1, \cdots v_{\mathrm{o}}$, which is used by the MLSE. The received signal samples are then down-sampled at the proper sampling instants and equalized by the MLSE. A flow-chart of the proposed algorithm is shown in Figure 2.

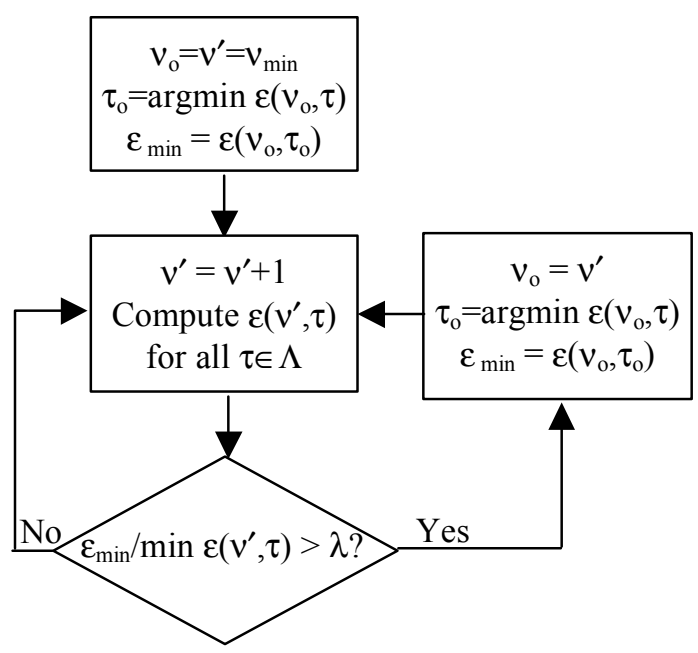

Figure 2. The flow chart of the proposed algorithm.
The proposed algorithm is justified as follows. It can be seen from (8) that since $\varepsilon\left(v^{\prime}, \tau\right)$ is a decreasing function of $v^{\prime}, \min \varepsilon\left(v^{\prime}, \tau\right)$ is also a decreasing function of $v^{\prime}$. Furtlfêmore, it is observed that, in general, $\left|\hat{\mathbf{x}}_{v^{\prime}}^{H} \mathbf{r}(\tau)\right|^{2}$ is also a decreasing function of $v^{\prime}$, and is typically very small when $v^{\prime}$ is greater than the true channel memory $v$. Therefore the ratio of $\min \varepsilon\left(v^{\prime}, \tau\right)$ to $\min \varepsilon\left(v^{\prime}+1, \tau\right)$ also in general decreases with $v^{\prime}$, and approacches 1 when $v^{\prime}>v$. In other words, the marginal improvement in the least-squares channel estimation error diminishes with $v^{\prime}$, and becomes very small when $v^{\prime}>v$. Therefore, in order to detect whether $v^{\prime}$ is sufficiently large, Step 4 of the proposed algorithm compares this marginal improvement in least-squares channel estimation error with a pre-determined threshold $\lambda$ and accepts a larger channel estimator memory only when the marginal improvement is greater than $\lambda$. This way $v_{o}$ is prevented from being too much larger than the true value $v$.

It should be noted that the main novelty of the proposed algorithm is that the parameters $\tau$ and $v^{\prime}$ are optimized jointly with the channel estimate. In a conventional least-squares channel estimator $\tau$ is usually separately optimized, and $v^{\prime}$ is usually preset to a fixed number. Furthermore, the minimization process is fairly simple because recursive computations are used, and involves only arithmetic operations that can be easily implemented using software or hardware.

\section{Simulation Results}

The performance of the proposed algorithm is evaluated by computer simulation. In each simulation, the transmitter is simulated according to the GPRS specifications as mentioned earlier. The simulated channel models include the hilly terrain (HT) and typical urban (TU) channels from the GPRS recommendations. No frequency offset or Doppler spread is assumed. Throughout the experiments the over-sampling factor $L$ is fixed at 4 . The feasible set $\Lambda$ for the sampling instant $\tau$ is $\Lambda=\left\{l T / L, l=l_{0}, l_{0}+1, \ldots, l_{0}+2 L\right\}$, where $l_{0}$ is a coarse sampling instant obtained using a previously proposed approach [4].

The average channel bit error rates (BER) of the MLSE using a conventional least-squares channel estimator with fixed length $v^{\prime}$ is shown in Figure 3 as functions of $v^{\prime}$. Here $E_{b} / N_{0}$ is fixed at $20 \mathrm{~dB}$, where $E_{b}$ is the transmitted energy per channel bit and $N_{0} / 2$ is the two-sided power spectral density of the AWGN. It can be seen from the figure that the optimal choices of $v^{\prime}$ for the TU and HT models are, respectively, 3 and 6. Therefore if $v^{\prime}$ is preset to 3, performance of the MLSE in the TU channel is optimized but the performance in the HT channel is greatly sacrificed. On the other hand, if $v^{\prime}$ is preset to 5 or 6 , both TU and HT performance will be acceptable. However, in a TU channel the equalizer is unnecessarily more complex because the complexity of the MLSE is exponentially related to $v^{\prime}$, and the performance is 


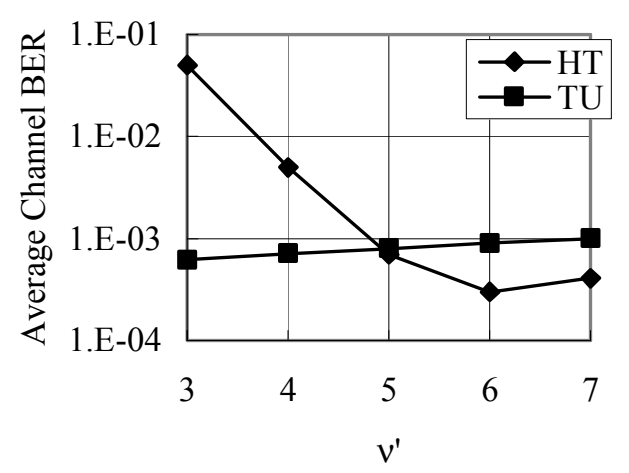

Figure 3. The performance of MLSE in the HT and TU channels using conventional least-squares channel estimators with different lengths.

slightly worse than when $\mathrm{v}^{\prime}$ is preset to 3 , as predicted previously[9]. It is therefore desirable to have a channel estimator that can, in principle and without explicit knowledge of the propagation environment, automatically set $v^{\prime}$ close to 3 when the receiver is operating in the TU environment and close to 6 when in the HT environment. This way the best trade-off between complexity and performance is achieved.

The performance of the proposed algorithm is shown in Figures 4 and 5 for the TU and HT channels, respectively. The decoded BER's of the CS-4 coding scheme are shown as functions of $E_{b} / N_{0}$ in these figures. Performance of the conventional least-squares channel estimator with $v^{\prime}$ preset to 3 (optimal for TU) and 6 (optimal for HT) are also shown as baselines for comparison. For the proposed algorithm, the receiver does not have prior knowledge of the channel condition (TU or HT), and the length of the channel estimator is not preset. An optimal channel estimator $v_{0}$ is chosen once per burst using the joint optimization described in the previous section. The threshold $\lambda$ is chosen to be 1.5 based on some preliminary simulations, and $v_{\min }=3$ and $v_{\max }=7$ is used based on the results in Figure 3.

It can be seen that the proposed algorithm achieves optimal performance in both channels. The performance loss with respect to the optimal channel estimator length is negligible. Detailed inspection of the simulation results shows that most of the time the proposed algorithm indeed automatically sets $v^{\prime}$ close to 3 for the TU channel and close to 6 for the HT channel. This observation confirms that the proposed algorithm is robust against propagation environment, and achieves the best trade-off between performance and complexity without explicit knowledge of the environment.

\section{CONCLUSION}

Equalization is an effective measure against ISI encountered in high-speed wireless data communication systems. For channel-estimation-based equalizers, the discrete-time equivalent channel is first estimated. The equalizer is then optimized based on the channel estimate. Conventional channel estimation algorithms preset a fixed length for the channel. For wireless communications, however, the actual length of the channel is environment-dependent. Therefore, unless the receiver has explicit knowledge of the propagation environment, the actual length of the wireless channel is unknown. Simulation results show that setting an optimistically small value for the length of the channel estimator greatly sacrifices the performance of the equalizer in malign channels, while setting the length of the channel estimator to a "worst-case" value may incur a performance loss and unnecessary equalizer complexity in benign environments. In this paper, we propose a new algorithm for joint synchronization, channel length estimation, and channel estimation. Simulation results show that the proposed scheme is robust against propagation environment and achieves the best trade-off between performance and complexity.

\section{REFERENCES}

[1] S. Qureshi, “Adaptive Equalization," Proceedings of IEEE, Vol. 73, No. 9, pp. 1349 - 1387, September 1985.

[2] P. K. Shukla et. al., "Channel-Estimation-Based adaptive DFE for Fading Multipath Radio Channels," IEE Proceedings-I, Vol. 138, No. 6, pp. 525 - 543, December 1991.

[3] R. A. Ziegler et. al., "Nonrecursive Adaptive DFE from Channel Estimates," IEEE Global Telecommunications Conference, pp. $600-603,1992$.

[4] Y. Lee and D. C. Cox, "Adaptive Equalization with Regularization for Indoor Wireless Data Communications," IEEE Global Telecommunications Conference, November 1997.

[5] D. Dzung, "Error Probability of MLSE Equalization Using Imperfect Channel Measurements," 1991 IEEE Internation Conf. on Communications, pp. 558 - 562.

[6] N. Seshadri, "Joint Data and Channel Estimation Using Blind Trellis Search Techniques," IEEE Trans. On Communications, Vol. 42, pp. 1000 - 1011, Feb./Mar./Apr. 1994.

[7] M. Stojanovic, J. G. Proakis, and J. A. Catipovic, "Analysis of the Impact of Channel Estimation Errors on the Performance of a Decision-Feedback Equalizer in Fading Multipath Channels," IEEE Trans. On Communications, Vol. 43, pp. 877 - 886, Feb./Mar./Apr. 1995.

[8] A. Gorokhov, "On the Performance of the Viterbi Equalizer in the Presence of Channel Estimation Errors," IEEE Signal Processing Letters, Vol. 5, No. 12, pp. 321 - 324, December 1998.

[9] Yumin Lee, Jyh-Hau Chen, "The Effect of Channel Estimator Memory Mismatch on the Performance of MLSE in Wireless Data Communications," 2002 IEEE International Conference on Communications, April 2002.

[10] GSM 05.03 version 7.2.0 Release 1998.

[11] Murota, K., and Hirade, K., "GMSK Modulation for Digital Mobile Radio Telephony," IEEE Transactions on Communications, vol. COM-29, no.7, pp.1044-1050, July 1981.

[12] Umberto Mengali, and Michele Morelli, "Decomposition of M-ary CPM Signals into PAM Waveforms," IEEE Trans. Commun. , vol. 41 , no.5, pp.1265-1275, Sep 1995.

[13] Simon Haykin, Adaptive Filter Theory, 3rd Ed., Prentice Hall, 1996. 


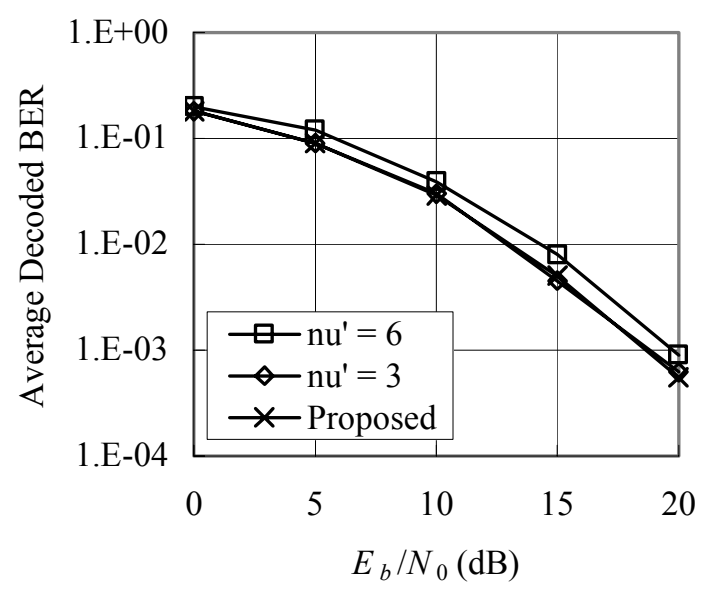

Figure 4. Average CS-4 decoded BER for the TU channel using the proposed algorithm and conventional channel estimator with $v^{\prime}=3$ and 6 .

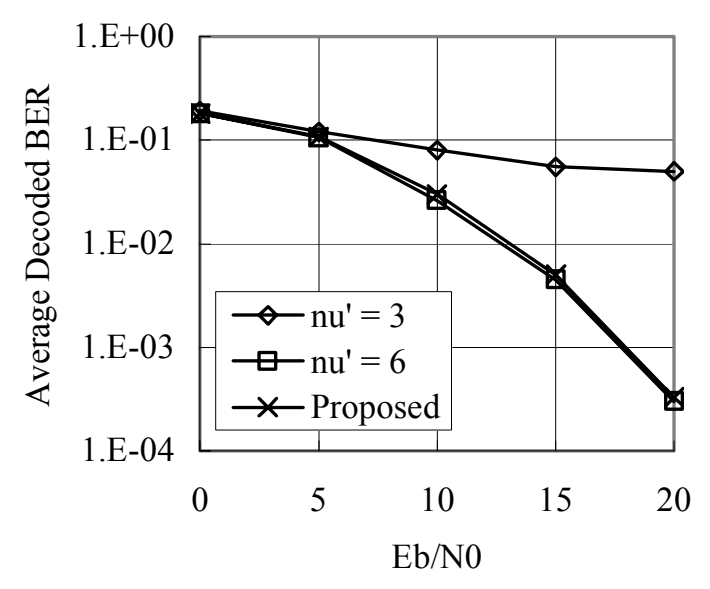

Figure 5. Average CS-4 decoded BER for the HT channel using the proposed algorithm and conventional channel estimator with $v^{\prime}=3$ and 6 . 